\title{
Lebendiges und Totes in Feuerbachs Straftheorie - Ein Beitrag zur gegenwärtigen strafrechtlichen Grundlagendiskussion
}

\author{
Tatjana Hörnle*
}

Rezension zu Luís Greco, Lebendiges und Totes in Feuerbachs Straftheorie. Ein Beitrag zur gegenwärtigen strafrechtlichen Grundlagendiskussion, Berlin (Duncker \& Humblot) 2009. 640 S., 118,00 €, ISBN 978-3-428-13008-5

Deutsche Abhandlungen zur Straftheorien beziehen sich häufig auf klassisch gewordene Texte aus dem 18. und 19. Jahrhundert. Typischerweise versuchen Anhänger der sog. absoluten Straftheorien Kriminalstrafe zu rechtfertigen, indem Passagen aus Schriften zitiert werden, die der Philosophie des deutschen Idealismus zugerechnet werden. Widmet sich ein Autor Feuerbachs Straftheorie, so könnte dies auf den ersten Blick den Verdacht wecken, dass er sich derselben Methode bedient und mittels ausführlicher Wiedergabe von Klassikerzitaten im Wettstreit von absoluten und relativen Straftheorien das Lager der relativen Straftheorien stärken will. Dieser Eindruck würde jedoch trügen. Die Schilderung der Feuerbachschen Lehre nimmt nur einen relativ kleinen Teil der von Luís Greco verfassten Monographie in Anspruch. Der Autor ist erfreulicherweise nicht der Ansicht, dass der Weg zu einer überzeugenden Straftheorie in der Auslegung eines historischen Textes liegt, sondern er ist ein analytisch denkender Wissenschaftler, der in systematischer Weise Argumente für und wider alle gängigen straftheoretischen Begründungsansätze untersucht. Im Ergebnis verteidigt er eine Androhungsgeneralprävention akzentuierende Straftheorie, aber mit Ergänzungen und Differenzierungen, die über die Gedanken und das Argumentationsniveau Feuerbachs weit hinausreichen.

Wer das Buch unter Auslassung des Vorwortes lesen würde, müsste davon ausgehen, dass es sich um eine Habilitationsschrift handele, und zwar eine gelungene Arbeit innerhalb dieser Literaturgattung (tatsächlich handelt es sich um die Dissertation eines jungen, aus Brasilien stammenden und seit einigen Jahren an der Ludwig-Maximilians-Universität München tätigen Wissenschaftlers; Doktorvater war Claus Roxin). Die hohe Qualität des Buches ist auf die Kombination mehrerer Umstände zurückzuführen. Greco ist mit einem außergewöhnlich breiten Spektrum wissenschaftlicher Literatur vertraut, was sich nicht nur im Literaturverzeichnis niederschlägt, sondern in einem genuin weiten Horizont beim Blick auf die Probleme. Dies gilt zum einen für die Art der verwerteten Literatur. Der Autor zieht rechtswissenschaftliche Schriften aus mehreren Jahrhunderten heran und er kennt die philosophischen Diskussionen. Zum anderen ist er einer der wenigen in der deutschen Strafrechtswissenschaft, der umfassend Material aus drei großen Sprachkreisen (das

* Prof. Dr. Tatjana Hörnle lehrt Strafrecht, Strafprozessrecht, Rechtsphilosophie und Rechtsvergleichung an der Humboldt-Universität zu Berlin. 
deutschsprachige, das spanisch-lateinamerikanische und das angloamerikanische Schrifttum) auswertet. Noch wichtiger: Das Buch bezeugt die souveräne Beherrschung des Umgangs mit rechtstheoretischen und rechtsphilosophischen, empirischen und normativen, konsequentialistischen und deontologischen Argumenten sowie das in intensiver Beschäftigung mit der Materie entwickelte Verständnis, dass eine überzeugende Straftheorie komplex und mehrdimensional ausfallen muss. Die Gedankenführung ist transparent, der Text flüssig und schnörkellos geschrieben.

Die Wahl des Themas könnte auf den ersten Blick die Frage provozieren, ob es für eine umfangreiche wissenschaftliche Publikation hinreichend Stoff liefere. Feuerbachs Ansatz wird in jedem noch so kurzen straftheoretischen Überblick erwähnt, und besonders kompliziert ist der zentrale Gedanke nicht, dass von Strafandrohungen im Gesetz eine psychologische Zwangswirkung ausgehe, die Straftaten verhindere. Zweifel an der Eignung des Themas sind jedoch schnell zerstreut. Dem Konzept der Androhungsgeneralprävention sind oft Argumente entgegengesetzt worden, die teils schnell zu widerlegen sind, teils aber eine sorgfältigere Auseinandersetzung verdienen. Greco arbeitet insbesondere sorgfältig heraus, an welchen Stellen eine funktionale, präventionsorientierte Herangehensweise nicht zu überzeugen vermag und welche deontologischen Grundsätze in ein Gesamtkonzept einzuarbeiten sind.

Das erste große Kapitel (überschrieben mit: rechtsgeschichtlicher Teil) enthält (S. 34-73) eine umfassende Beschreibung der Lehre Feuerbachs, nicht nur beschränkt auf straftheoretische Überlegungen im engeren Sinn, sondern auch zum Verbrechen als Rechtsverletzung, zum Gesetzlichkeitsprinzip, zur Strafzumessung und zum Strafvollzug. Dabei legt er auch illiberale Züge Feuerbachs offen, etwa die Befürwortung von Körperstrafen, entehrenden Strafen und der Todesstrafe. Greco erörtert sodann, in welchem Verhältnis Feuerbachs nüchtern-zweckrationaler Verweis auf den „psychologischen Zwang “ zur Straftheorie Kants steht, was erklärungsbedürftig sei, weil Feuerbach sich als Kantianer verstanden habe. Letztlich kommt Greco zu einem salomonischen (und durchaus plausiblen) Urteil: Das Gesamtwerk Kants bietet Anknüpfungspunkte für unterschiedliche straftheoretische Ausarbeitungen (S. 87). Im folgenden Abschnitt (S. 87-107) räumt Greco mit einem Vorurteil auf, nämlich dem Vorurteil, dass Feuerbach ein übertrieben rationalistisches Menschenbild zugrunde gelegt habe. Er zeigt, dass Feuerbachs Vorstellung vom „psychologischen Zwang“ nicht den „homo oeconomicus“ voraussetze, sondern, assoziationspsychologisch, Furcht als Antrieb.

Das zweite Großkapitel trägt die Überschrift „rechtsphilosophischer Teil“. Solche Überschriften wecken bei der Rezensentin aus einschlägiger Leseerfahrung die Befürchtung, dass unkritisch-bekenntnisartige Deklamationen oder eine Flut von Exzerpten aus den Texten philosophischer Klassiker folgen könnten. Die Überlegungen, die Greco anstellt, beruhen jedoch auf seinem eigenständigen, analytischen Zugang 
zu einer rechtsphilosophischen Grundlagenfrage: Er setzt sich mit dem Postulat der Trennung von Recht und Moral auseinander, und zwar ausgehend von den (S. 109-117) skizzierten Gefahren eines moralisierenden Strafrechts einerseits, eines strafrechtlichen Amoralismus (in totalitären Systemen) andererseits. Er sieht die Notwendigkeit einer nicht-konsequentialistischen, deontologischen Moral (eine zentrale Rolle spielt dabei das Verbot von Folter), will aber „side constraints“ oder Schranken bei der Verfolgung von Zwecken auf das Handeln von Staatsorganen beschränken. Im Verhältnis zum Bürger lehnt er mit guten Gründen die mit Zwang verbundene Verhaltensregulierung zur Sicherung tugendethischer Vorstellungen und deontologisch begründeter Normen ab (S. 123-143). Charakteristisch für Grecos Arbeit sind Exkurse, die Grundsatzfragen vertiefen, so etwa zum Thema Nonkognitivismus, eine metaethische Position, die Greco ablehnt (S. 143-160). Solche Exkurse passen zu einer Herangehensweise, die auch die Prämissen eines Arguments (etwa: ob man überhaupt in sinnvoller Weise über ethische Thesen diskutieren kann) hinterfragt. Inhaltlich scheint mir fraglich, ob Greco nicht den Kreis derer, die er bei den Nonkognitivisten einordnet (s. die Nwe. in Fn. 125) zu weit zieht oder jedenfalls eine solche Einordnung überbewertet. Nicht jeder, der Letztbegründungen für unmöglich hält, muss folgern, dass ethische Diskurse keinen Erkenntniswert haben.

Der nächste Abschnitt „der Mensch als Zweck an sich selbst“ (S. 160-202) führt wieder näher zu Feuerbach zurück, weil ein zentraler Einwand gegen dessen generalpräventive Straftheorie lautet, dass diese Menschen als Objekt behandle, in anderen Worten: sie instrumentalisiere. Greco kämpft mit dem Problem, das Instrumentalisierungsverbot zu präzisieren. In diesem Ringen um Begründungen liegt eine wissenschaftliche Qualität, die sich vor allem im Kontrast zu Arbeiten zeigt, die weitreichende Schlussfolgerungen leichthändig aus einer Fundstelle in einem Klassikertext deduzieren. Greco nennt (als „Versuch“ bezeichnet) einige absolute, deontologische Verbote (S. 180-190), etwa das Verbot der Todesstrafe, der Folter, aber auch der lebenslangen Freiheitsstrafe. Von solchen Instrumentalisierungen i.e.S. unterscheidet er Instrumentalisierung i.w.S., d.h. Handeln gegen den Willen des Betroffenen, das nicht absolut verboten sein kann, aber eine Begründungslast schafft (S. 191 f.). Die Unterscheidung von Instrumentalisierung i.e.S. und i.w.S. ist wichtig (und überzeugend), weil sie erlaubt, den Standardeinwand gegen negative Generalprävention wegen des zu pauschalen Verweises auf Instrumentalisierung zurückzuweisen.

Im dritten und längsten Teil der Arbeit (dem strafrechtsphilosophischen Teil) widmet sich Greco zunächst abolitionistischen Fundamentaleinwänden, die er kategorisiert und widerlegt (S. 207-226). Dann begründet er, warum zwischen Androhung und Verhängung von Strafen unterschieden werden muss (S. 228 f.; weil es sich um unterschiedliche Freiheitseingriffe handelt). Es folgt eine zentrale wie überzeugende 
Festlegung: Eine Straftheorie kann weder nur konsequentialistisch noch nur mit dem Verweis auf Gerechtigkeit begründet werden (S. 230-234). Genauso wenig trägt der beliebte Verweis auf „Prävention durch Gerechtigkeit“ (S. 234-247), weil eine nur instrumentelle Begründung von Gerechtigkeit zwar oft zum gewünschten Ergebnis führt, aber genau dann versagt, wenn es zu einem Konflikt mit präventiver Effizienz kommt. Es bedarf, hier stimme ich mit Greco uneingeschränkt überein, einer materiell zweigeteilten Straftheorie. Zu differenzieren ist zwischen Strafzwecken, die zweckbezogen, also konsequentialistisch, sind, und deontologischen Schranken, also ausnahmslos geltenden Regeln, die die Verfolgung des Strafzwecks beschränken. In der Literatur wird dem entgegengehalten, dass „axiologische Geschlossenheit“ erforderlich sei (so Pawlik, Person, Subjekt, Bürger, 2004, S. 53). Greco führt diese Sehnsucht nach Einheitlichkeit auf ästhetische Motive zurück (eine Vermutung, die ich auch hege), er verweist aber mit Roxin darauf, dass man damit der Komplexität der Phänomene nicht gerecht werde (S. 252).

Einen Exkurs zur Strafgesetzlichkeit nutzt Greco, um auch insoweit zu zeigen, dass die konsequentialistische Begründung, die Feuerbach bevorzugte, eine fragile Begründung ist (was sich zeigt, wenn wie im Nationalsozialismus Abschreckung ernst genommen wird) und man das Gesetzlichkeitsprinzip deshalb besser als intrinsisch wichtig und als deontologische Schranke verstehen sollte (S. 253-274). Zurück zur Straftheorie, folgt eine einleuchtende Begründung, dass der Begriff oder die Definition von Strafe von ihrer Rechtfertigung unterschieden werden sollte (S. 274-303), was zur Definition von Strafe als „vom Staat verhängte, besonders schwere Übel körperlicher oder kommunikativer Art, die als objektive Reaktion für eine angenommene Straftat verhängt werden“ führt (S. 303). Im folgenden Abschnitt geht Greco ausführlich auf Kriminalisierung ein, d.h. auf die Frage, ob Strafnormen Rechtsgüter oder subjektive Rechte schützen (S. 303-354). In diesem Zusammenhang relativiert er unter anderem allzu optimistische Assoziationen, die mit der geschichtlichen Periode der Aufklärung verbunden werden. Im Ergebnis stimme ich dem Verf. nicht zu, der meint, dass „Rechtsgüter“ zur Bezeichnung der strafrechtlichen Schutzobjekte vorzugwürdig sei. Zwar kommt es, wie Greco ausführt (S. 333), auf Kontinuitäten oder Diskontinuitäten mit historischen Schriften nicht an, aber ich halte daran fest, dass zu einem im Verhältnis zu Individuen nicht moralistischen Strafrecht besser die Fokussierung auf „Rechte anderer“ passt als das kriminalpolitisch beliebig einsetzbare Argument „Schutz eines Rechtsgutes“.

Es folgt die Auseinandersetzung mit dem bekanntesten Element der Feuerbachschen Lehre: dem Konzept der psychologischen Abschreckung. Greco kritisiert hier den psychologischen Zuschnitt, d.h. die Konzentration auf das Motiv Furcht. Er spricht sich für einen funktionalen Abschreckungsbegriff aus, der auf Gründe, nämlich Klugheitsgründe setzt (S. 356-362). Der erste, immer wieder erhobene Einwand ge- 
gen die These, dass Menschen sich durch solche Gründe beeinflussen lassen, bezieht sich auf empirische Zusammenhänge. Greco geht nur relativ knapp auf empirische Untersuchungen ein (S.363-365), weil er meint, es offen lassen zu können, wer von den Wissenschaftlern, die überwiegend Abschreckungswirkung bejahen, teilweise aber auch verneinen, Recht habe. Juristen könnten sich für einen „gesunden Allgemeinsinn " entscheiden (S. 365-369). In dieser methodischen Frage, zum Verhältnis von Rechts- und Sozialwissenschaften, würde ich ihm widersprechen - aber immerhin reflektiert Greco, ob die Option für den „alltäglichen Realismus“ vertretbar ist (was viele Rechtswissenschaftler nicht tun). Im Ergebnis geht er davon aus, dass Gründe zu Motiven werden können - nicht immer und nicht bei allen Menschen, aber das setzt die These von der Androhungsgeneralprävention auch nicht voraus, was Greco gegenüber anders lautenden Stimmen verdeutlicht (S. 369-377).

Bedeutsam ist die Auseinandersetzung mit den normativen Einwänden, die gegen die Theorie von der Abschreckungsgeneralprävention vorgebracht werden, genauer, gegen deren Prämissen und Folgen. Die erste Kategorie von Bedenken betrifft den bereits widerlegten Instrumentalisierungsvorwurf und das Menschenbild (Hegels Vergleich mit dem Hund, gegen den der Stock erhoben wird). Greco verweist auf seine funktionale Reformulierung: Nicht primäre Furcht ist ausschlaggebend, sondern die Fähigkeit des Menschen, Klugheitsregeln zu verstehen (S. 379-381). Zur zweiten Kategorie der Einwände gehört der Verweis auf die Gefahr disproportionaler Strafen. Mit dem Argument, dass Strafzweckerwägungen durch deontologische Schranken (wozu die Proportionalität der Strafe gehört) eingeschränkt werden, ist dieser Einwand zu entkräften (S. 381-389). Ebenso setzt Greco dem vielfach zitierten Argument Jakobs', dass es auf den Tatnutzen ankomme (der bei einem Mord geringer sein könne als bei einer Verleumdung) ein Gegenargument entgegen: Verhaltenssteuerung durch Normen kann nur generell und nicht bezogen auf konkrete Tatvorteile angestrebt werden (S. 389 f.).

In weiteren Passagen erörtert der Verf. andere straftheoretische Ansätze. Im Abschnitt zur positiven Generalprävention als Strafandrohungsgrund zeigt sich Greco kritisch. Er hält es für unzulässig, moralische Verhaltensanforderungen zu verdeutlichen (zu erziehen), wenn dies mit Zwang geschehe, dies mit Kritik an „volkserziehenden “ Ansätzen im 19. und 20. Jahrhunderts (S. 396-419). Grecos Argument bedarf der Diskussion. Es lebt von der Suggestivkraft von Begriffen wie „Erziehung“ und „Zwang“ und dem Verweis auf die nicht-rechtsstaatlichen Hintergründe der zitierten Autoren. Strafnormen kann man aber in einem modernen Rechtsstaat als Appell, verbunden mit einer Klugheitsregel, verstehen. Die Vorstellung, dass der Staat der Bereitschaft der Bürger, rechtlich kodifizierte Verhaltensnormen zu akzeptieren, grundsätzlich indifferent gegenüber stehen müsse, scheint mir eher abwegig. Genauso wenig überzeugt mich das Argument, dass die Verhängung von Strafen zur 
Normverdeutlichung in die Freiheit aller Bürger eingreife (S. 457) - die expressive Botschaft bedeutet keine Freiheitseinbuße für diejenigen, die nach der Theorie der positiven Generalprävention Erhaltung ihrer Orientierungssicherheit wünschen. Eine Entscheidung zwischen entweder negative oder positive Generalprävention halte ich im Gegensatz zu Greco nicht für erforderlich - beide Aspekte gehören zu einer zweckrationalen Straftheorie.

Auf die Frage, warum Strafen nicht nur angedroht, sondern ggf. auch verhängt werden müssen, antwortet Greco knapp entsprechend der Feuerbachschen Vorgabe, dass ansonsten die Androhung nicht mehr ernst genommen werde. Allerdings erkennt er, dass dieser Strafzweck nicht unbedingt erfordere, dass auf alle Normübertretungen reagiert wird (S. 420-422). Der Verf. legt dann zugrunde, dass das Legalitätsprinzip gelten müsse, und zwar nicht konsequentialistisch begründet, sondern als „prozessuale Kehrseite des materiellrechtlichen Gesetzlichkeitsprinzips“ (S. 423). An dieser Stelle wäre größerer Begründungsaufwand erforderlich: Es ist nicht selbstverständlich, dass eine strikte Pflicht zur Strafverfolgung unverzichtbar ist. Von seiner Prämisse ausgehend, macht Greco sich Gedanken über die Umsetzung des Legalitätsprinzips in modernen Strafrechtsordnungen und spricht sich unter anderem für Entkriminalisierungen aus (S. 424-434). Unschädlichmachung und Besserung als Gründe für Strafverhängung lehnt der Verf. ab (S. 435-453). Für Strafverhängung ist, anders als beim bloßen Appell der Strafnorm, wegen des ausgeprägten Grundrechtseingriffs in der Tat gut zu begründen, dass dies nicht mit erzieherischen Anliegen zu legitimieren ist. Überwiegend Zustimmung verdienen Grecos Ausführungen $\mathrm{zu}$ Vergeltungstheorien, die er in Unterkategorien aufgliedert und allesamt kritisch beurteilt (S. 458-477). Allerdings möchte ich einwenden, dass die Zusammenfassung von deontologischen und konsequentialistischen Ansätzen unter der Gesamtüberschrift „Vergeltung “ erhebliche Unterschiede überspielt. Einige der Theorien, die der Strafe einen Zweck zumessen, sollte man besser als expressive oder kommunikationsorientierte Straftheorien bezeichnen und klarer von zweckfreier Vergeltung abgrenzen. Derartige Ansätze, die in den letzten Jahren zunehmend Anhänger finden, werden vom Verf. erwähnt, aber nicht ausführlich gewürdigt. Insbesondere gilt dies für Überlegungen, dass der Zweck von Strafe bei schwerwiegenden Taten gegen Personen darin liegen könnte (nach Auffassung der Rezensentin: darin liegen muss), berechtigten Genugtuungsinteressen der Opfer zu genügen.

Interessant sind die Überlegungen zum Rechtsgrund der Strafzufügung, d.h. zu der Frage, ob die Verhängung einer Strafe gegenüber dem Bestraften legitim ist (S. 484-509). Greco versucht hier, aus der - zu Recht als unhaltbar eingestuften Einwilligungsthese Feuerbachs einen Grundgedanken zu retten, der im Gegensatz zu verbreiteten Schuldkonzepten steht. Sein Anliegen ist, wie auch an anderen Stellen der Arbeit, eine konsequente Entmoralisierung von staatlichen Bewertungen des 
Verhaltens der Bürger. Demgemäß definiert Greco Schuld als „strafbezogene Unklugheit" (S. 490) und wendet sich gegen die Vorstellung, dass das Strafurteil mit einer mehr als nur deklaratorischen Missbilligung verbunden sei (S. 501). Damit macht er jedoch meines Erachtens das unmöglich, was er anstrebt, nämlich die Verhängung von Strafe gegenüber den Bestraften zu legitimieren. Unklugheit ist eine Wertung, die ausschließlich das Verhältnis des Straftäters zu sich selbst, die Zerstörung seiner eigenen Lebensplanung (s. dazu S. 491-493), thematisiert. Strafe als von außen kommender Eingriff kann jedoch nur legitimiert werden, wenn man dem Täter einen Fehler vorwerfen kann, den er im Verhältnis zu anderen gemacht hat (etwa, dass er als „Trittbrettfahrer“ von der Normkonformität anderer profitiere, ohne sich selbst zu beschränken).

Selbst mit dieser relativ langen Besprechung sind nicht alle Gedanken gewürdigt, die in Grecos Arbeit stecken. Dass es bei einer so gehaltvollen und thesenreichen Untersuchung jedenfalls an einigen Stellen zu einem inhaltlichen Dissens zwischen Leser und Autor kommen muss, ist unvermeidbar. Den Wert der wissenschaftlichen Arbeit und der anregenden Lektüre schmälert dies nicht. Hervorzuheben ist abschließend, dass die von Greco immer wieder betonte Differenzierung zwischen zweckorientierten Überlegungen und deontologischen Schranken es erlaubt, ein differenzierteres Bild einer modernen Straftheorie zu zeichnen als der verbreitete, aber schwammige Verweis auf eine „Vereinigungstheorie“, die absolute und relative Straftheorien vereinigen soll. 